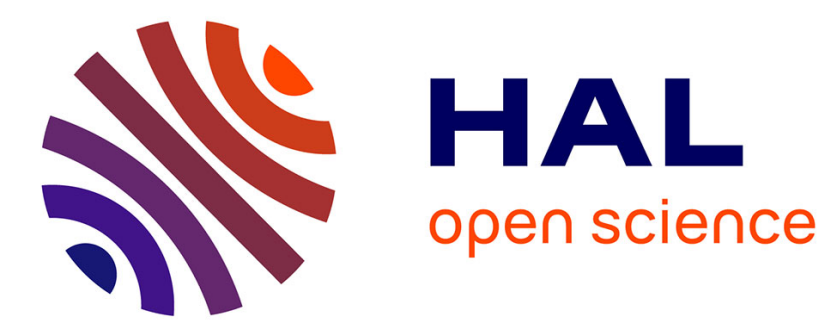

\title{
Polarisation de charge (ou de spin) au voisinage d'une impureté dans un alliage
}

P.-G. de Gennes

\section{To cite this version:}

P.-G. de Gennes. Polarisation de charge (ou de spin) au voisinage d'une impureté dans un alliage. J. Phys. Radium, 1962, 23 (10), pp.630-636. 10.1051/jphysrad:019620023010063000 . jpa-00236651

\section{HAL Id: jpa-00236651 https://hal.science/jpa-00236651}

Submitted on 1 Jan 1962

HAL is a multi-disciplinary open access archive for the deposit and dissemination of scientific research documents, whether they are published or not. The documents may come from teaching and research institutions in France or abroad, or from public or private research centers.
L'archive ouverte pluridisciplinaire HAL, est destinée au dépôt et à la diffusion de documents scientifiques de niveau recherche, publiés ou non, émanant des établissements d'enseignement et de recherche français ou étrangers, des laboratoires publics ou privés. 


\title{
POLARISATION DE CHARGE (OU DE SPIN) AU VOISINAGE D 'UNE IMPURETÉ DANS UN ALLIAGE
}

\author{
Par P. G. DE GENNES,
}

C. E. N. de Saclay et Faculté des Sciences, Orsay.

\begin{abstract}
Résumé. - On calcule la polarisation de charge (ou de spin) induite en un point $B$ dans un alliage au voisinage d'une impureté A en tenant compte de la diffusion des électrons par d'autres impuretés (réparties au hasard) entre A et B. Les applications possibles de ce calcul comprennent : 1) La discussion des facteurs de forme magnétiques d'impuretés (ceux-ci étant nécessairement mesurés à des concentrations relativement élevées).2) L'étude des transformations ordre désordre : dans un alliage subissant une telle transformation, le libre parcours moyen varie fortement avec la température, et l'interaction effective entre atomes également. 3) L'étude des configurations magnétiques des métaux de terres rares, où le même mécanisme pourrait expliquer la variation du pas des hélices avec la température. 4) La disparition de l'anomalie de Kohn dans les spectres de phonons en présence de diffusion par des impuretés ou par les vibrarions de réseau.
\end{abstract}

\begin{abstract}
The charge (or spin) polarisation induced in its vicinity by an impurity A at point B in an alloy is calculated with inclusion of possible scattering effects by other impurities distributed at random between A and B. Possible applications of the calculation include : 1) The question of the form factors of magnetic impurities (these being necessarily measured at relatively high concentrations. 2) Order disorder transformations : in an alloy undergoing such a transformation, the mean free path varies strongly with temperature and the effective interaction between atoms becomes also temperature dependent. 3) Magnetic order in the rare earth metals : the observed change of pitch of the spirals with temperature having possibly the same origin. 4) The suppression of the Kohn anomaly in phonon spectra due to impurity (or thermal) scattering of the electrons.
\end{abstract}

\section{INTRODUCTION.}

Considérons un spin nucléaire $I_{0}$ couplé par une interaction hyperfine $A \boldsymbol{I}_{0} S_{i} \delta\left(r_{i}-r_{0}\right)$ aux électrons de conduction (de spin $S_{i}$, de coordonnée $r_{i}$ ) d'un métal. En présence de $I_{0}$ les électrons développent une polarisation de spin

$$
\begin{aligned}
P(r) & =\sum_{i} s_{I} \delta\left(r-r_{i}\right) \\
& =-\frac{A \boldsymbol{I}_{0}}{4} \chi\left(r-r_{0}\right) .
\end{aligned}
$$

La susceptibilité locale $\chi(r)$ ainsi définie a été calculée pour un métal pur dans l'approximation des électrons libres par Ruderman et Kittel [1]

$$
\chi(r)=-\frac{m}{(2 \pi)^{3} \hbar^{2}} \frac{1}{r^{4}}\{2 \rho \cos 2 \rho-\sin 2 \rho\} .
$$

Dans cette formule $k_{F}$ est le vecteur d'ondes au niveau de Fermi, $\rho=k_{F} r$ et $m$ la masse des électrons. Plus généralement, quand $\chi(r)$ est connu, on peut calculer à une bonne approximation tous les effets de polarisation (de spin ou de charge) induits dans le métal par une faible perturbation statique, de répartition spatiale quelconque. Par exemple, la constante diélectrique $\varepsilon(q)$ du gaz d'électrons pour une perturbation statique sinusoïdale de longueur d'onde $2 \pi / q$ est donnée dans l'approximation de la phase aléatoire [2] par :

$$
\begin{aligned}
\varepsilon(p) & =1+\frac{4 \pi e^{2}}{p^{2}} \chi(p) \\
\chi(p) & =\int \mathrm{d}_{3} r \mathrm{e}^{i p . r} \chi(r) .
\end{aligned}
$$

Le but du présent article est d'étendre le calcul de $\chi(r)$ ou $\chi(p)$ aux métaux impurs. (Dans ce cas, on définit $\chi(r)$ comme une valeur moyenne prise sur toutes les configurations possibles des impuretés dans le métal.) Une fois ce programme réalisé, on peut notamment prendre comme origine de la perturbation une impureté A de position fixée dans l'alliage, et calculer la polarisation de charge ou de spin due à celle-ci, en présence de toutes les autres impuretés réparties au hasard. Ceci représente une deuxième étape dans la méthode de Friedel [3], la première étape revenant à considérer l'effet de A dans la matrice pure.

La "susceptibilité généralisée " $\chi(r)$ diffère de sa valeur dans le métal pur $\chi_{p}(r)$ pour deux raisons qui peuvent être clairement distinguées dans les alliages dilués : 1) il y a un déplacement des niveaux à un électron ; 2) les états d'onde plane acquièrent une durée de vie finie, correspondant à un libre parcours moyen $l$ également fini. Ces deux effets sont liés respectivement au comportement de la partie réelle et de la partie imaginaire de la matrice de 
diffusion par une impureté. Le premier peut être important numériquement mais est relativement trivial; dans toute la suite de cet article nous l'éliminerons systématiquement. (En particulier la densité de niveaux à l'énergie de Fermi sera supposée égale à sa valeur dans le métal pur.) Par contre, les effets liés à l'existence d'un libre parcours moyen fini $l$ sont plus intéressants et également plus complexes.

Une erreur à ne pas commettre consisterait à utiliser la formule "intuitive »

$$
\chi(r)=\chi_{p}(r) \mathrm{e}^{-r \boldsymbol{r}} \text {. }
$$

Même lorsque le libre parcours moyen $l$ est indépendant de l'énergie, l'équation (5) n'est pas correcte. Ceci peut être déduit de la règle de somme

$$
\int \chi(r) d_{3} r=\int \chi_{p}(r) d_{3} r
$$

qui exprime que la susceptibilité globale pour une perturbation homogène est la même dans l'alliage et dans le métal pur (puisque nous imposons la même densité de niveaux dans les deux cas). L'équation (6) n'est pas en général compatible avec (5) et (5) doit donc être rejetée. Nous verrons en fait que l'effet des impuretés n'est pas une simple atténuation de $\chi_{p}(r)$, mais implique aussi un changement de phase des oscillations exhibées par l'équation (2).

Les formules générales donnant $\chi(r)$ peuvent être établies à la seule condition que les impuretés soient diluées ( $l$ très supérieur à la distance entre impuretés et à la portée du potentiel perturbateur dû à une impureté). Elles ne peuvent être obtenues que par une théorie assez fine de la diffusion multiple, dont les bases ont été jetées par Edwards et Abrikosov et Gor'kov [4]. Pour le problème particulier qui nous intéresse, il faut notamment tenir compte très soigneusement des effets du " milieu " (constitué par les autres impuretés) dans chaque processus élémentaire de diffusion; sinon on rencontre des divergences parasites dans le développement de diffusion multiple. A ce détail près, la méthode de calcul est classique et suit très exactement les"procédés de la réf. [4]. Pour obtenir des résultats calculables en pratique, toutefois, nous nous limiterons au cas simple suivant : diffusion par les impuretés traitée seulement dans l'approximation de Born, et libre parcours moyen supposé indépendant de l'énergie.

\section{CALCUL DE $\chi(r)$}

1. Expression de $\chi$ en termes de fonctions de Green. - Nous considérons un métal impur, l'énergie d'un électron étant de la forme

$$
\mathcal{H}_{U} \cdot \overrightarrow{\nabla^{2}}=-\frac{\nabla^{2}}{2 m}+W(r)+U(r)
$$

$p^{2} / 2 m$ est le terme cinétique, $W(r)=\sum_{i} V\left(r-r_{1}\right)$ est le potentiel dû aux impuretés, et $U(r)=U_{0} \delta_{3}(r)$ représente la petite perturbation locale qui sert à définir $\chi(r)$ [5]. (Dans toute cette section, nous faisons $\hbar=1$ ). La densité d'électrons (d'une direction de spin) induite par la perturbation $U$ vaut

$-U_{0} \frac{\chi(r)}{2}=\langle\overline{U|\psi+(r) \psi(r)| U}>-<\overline{0|\psi+(r) \psi(r)| 0}>$

où $\mid U>$ et $\mid 0>$ représentent l'état de base du système à $N$ électrons respectivement en présence et en l'absence de la perturbation $U$, et $\psi(1)$ est l'opérateur habituel d'annihilation. Le symbole représente une moyenne sur toutes les configurations $\left(r_{1}\right)$ des impuretés supposées réparties au hasard. Nous avons omis les indices de spin qui n'interviennent pas dans le calcul.

Nous allons transformer (8) en introduisant trois fonctions de Green $G^{P}, G, G^{D}$, à un électron correspondant respectivement au métal pur $(W=0)$ au métal impur, mais sans la perturbation $U$, et au métal impur perturbé décrit par (7). Chacune de ces fonctions est définie par une équation de la forme

$$
G\left(r r^{\prime} t\right)=i<\left|T \psi(r t) \psi+\left(r^{\prime} 0\right)\right|>
$$

où $T$ est l'opérateur chronologique habituel et $\mid>$ l'état de base correspondant au cas considéré. Nous utiliserons surtout les transformées de Fourier, définies par

$$
\frac{1}{2 \pi} \int G_{\omega}\left(r r^{\prime}\right) \mathrm{e}^{-i \omega t} \mathrm{~d} t=G\left(r r^{\prime} t\right)
$$

qui satisfont aux équations

$$
\left.\begin{array}{l}
\left(-\frac{\hbar^{2}}{2 m} \nabla_{r}^{2}+W+U-\hbar \omega\right) G_{\omega}^{U}\left(r r^{\prime}\right)=\delta_{3}\left(r-r^{\prime}\right) \\
\left(-\frac{\hbar^{2}}{2 m} \nabla_{r}^{2}+W\right) G_{\omega}\left(r r^{\prime}\right)=\delta_{3}\left(r-r^{\prime}\right) \\
-\frac{\hbar^{2}}{2 m} \nabla_{r}^{2} G_{\omega}^{P}\left(r r^{\prime}\right)=\delta_{3}\left(r-r^{\prime}\right)
\end{array}\right\}
$$

ou sous forme intégrale

$$
\begin{aligned}
& G_{\omega}^{U}\left(r r^{\prime}\right)=G_{\omega}\left(r r^{\prime}\right)-\int G_{\omega}(r r) U(S) G_{\omega}^{U}\left(S r^{\prime}\right) \mathrm{d}_{3} S \\
& G_{\omega}\left(r r^{\prime}\right)=G_{\omega}^{P}\left(r r^{\prime}\right)-\int G_{\omega}^{P}\left(r r^{\prime}\right) W(S) G_{\omega}\left(S r^{\prime}\right) \mathrm{d}_{3} S .
\end{aligned}
$$

En comparant (8) et (9) on peut écrire

$$
\begin{aligned}
-U_{0} \frac{\chi(r)}{2} & =i[\overline{G U(r 0, t=-0)}-\overline{G(r 0, t=-0)}] \\
& =\frac{i}{2 \pi} \int \mathrm{d} \omega\left[G_{\omega}^{U}(r 0)-G_{\omega}(r 0) \mathrm{e}^{-i \omega t}\right](t=-0)
\end{aligned}
$$

En utilisant (12) et en se limitant au premier ordre en $U$, ceci peut s'écrire

$\chi(r)=\frac{i}{\pi} \int \mathrm{d} \omega \mathrm{e}^{-i \omega t} \quad \overline{G_{\omega}(r 0) G_{\omega}(0 r)} \quad(t=-0)$. 
2. Calcul des moyennes. - Il nous reste à calculer la moyenne du produit de deux fonctions de Green qui figure dans (14). Une erreur à éviter consisterait à écrire $\overline{G G} \cong \bar{G} \bar{G}$. Une telle approximation est totalement injustifiée. (Elle conduirait d'ailleurs à la formule inexacte (5).)

Pour des impuretés diluées, de concentration $n$, traitées dans l'approximation de Born, les méthodes de la référence [4] nous permettent de calculer des moyennes de la forme

$$
\overline{G_{\omega}\left(r r^{\prime}\right)} \text { et } F_{\omega}\left(r r^{\prime}\right)=\overline{G_{\omega}(r 0) G_{\omega}\left(0 r^{\prime}\right)}
$$

en utilisant les équations intégrales approchées [6] :

$$
\begin{aligned}
& \overline{G_{\omega}\left(r r^{\prime}\right)}=G_{\omega}^{0}\left(r r^{\prime}\right) \\
& +\int \mathrm{d}_{3} S \mathrm{~d}_{3} t G_{\omega}^{0}(2 S) \overline{W(S) W(t)} \overline{G_{\omega}(S t)} \overline{G_{\omega}\left(t r^{\prime}\right)} \\
& F_{\omega}\left(r r^{\prime}\right)=\overline{G_{\omega}(r 0)} \overline{G_{\omega}\left(0 r^{\prime}\right)} \\
& +\int \mathrm{d}_{3} S \mathrm{~d}_{3} t \overline{G_{\omega}(r S)} \overline{G_{\omega}\left(t r^{\prime}\right)} \overline{W(S) W(t)} F_{\omega}(S t) .
\end{aligned}
$$

On peut vérifier que, en ce qui concerne la partie longue distance de $\chi(r)$, le choix de l'équation approchée (15) revient à sommer les diagrammes les plus divergents d'un développement en perturbation [4]. Aux courtes distances, les diagrammes inclus dans (-15) ne sont pas particulièrement importants par rapport aux diagrammes omis, mais dans ce domaine le développement en perturbation converge très rapidement et la sélection s'effectue entre des diagrammes d'ordre élevé qui ' sont de toute façon négligeables.

3. Structure de la fonction $\bar{G}$. Étudions tout d'abord la structure de la moyenne simple $\overline{G\left(r r^{\prime}\right)}$.

Posons :

$$
\begin{aligned}
\overline{G_{\omega}(k)} & =\int \mathrm{d}_{3} r \overline{G_{\omega}(0 r)} \mathrm{e}^{i k r} \\
V(k) & =\int V(r) \mathrm{d}_{3} r \mathrm{e}^{i k r} .
\end{aligned}
$$

Dans le métal pur, on a

$$
G_{\omega}^{0}(k)=\frac{1}{\xi_{k}-\omega-i \varepsilon\left(\xi_{k k}\right)}
$$

où $\xi_{k}=\frac{k^{2}}{2 m}$ et $\varepsilon\left(\xi_{k}\right)$ est un infiniment petit du signe de $\xi_{k}-\frac{k_{\mathrm{F}}^{2}}{2 m}$.

L'équation (14) peut se récrire

$$
\begin{aligned}
\overline{G_{\omega}(k)}=G_{\omega}^{0}(k) & {\left[1+n(2 \pi)-^{3}\right.} \\
\times & \int \mathrm{d}_{3} k^{\prime}\left|V\left(k-k^{\prime}\right)\right|^{2} \overline{G_{\omega}\left(k^{\prime}\right)} \overline{G_{\omega}(k)} \\
& =\frac{1}{\xi_{k}-\omega+\Delta_{k}(\omega)}
\end{aligned}
$$

où $\Delta_{\boldsymbol{k}}$ représente une correction (complexe) à l'énergie de l'état $k$

$$
\Delta_{k}(\omega)=-n(2 \pi)^{-3} \int \mathrm{d}_{3} k^{\prime}\left|V\left(k-k^{\prime}\right)\right|^{2} \overline{G_{\omega}\left(k^{\prime}\right)} .
$$

Dans la suite, nous supposerons pour simplifier que $V(k)$ est indépendant de $k, V(k)=V$ (ce qui conduit, en particulier, à un libre parcours moyen indépendant de l'énergie). Dans ce cas $\Delta_{k}(\omega)$ est fonction seulement de $\omega$ et on peut résoudre explicitement (20). Écrivons

$$
\begin{gathered}
\Delta(\omega)=\Delta_{0}-n(2 \pi)^{-3} \int \mathrm{d}_{3} k^{\prime}\left(\frac{1}{\xi_{k^{\prime}}-\omega+\Delta(\omega)}-\frac{1}{\xi_{k}}\right) \\
\Delta_{0}=-n V^{2}(2 \pi)^{-3} \int \mathrm{d}_{3} k^{\prime} \frac{1}{\xi_{k^{\prime}}}
\end{gathered}
$$

$\Delta_{0}$ représente le déplacement en énergie du bas de la bande, dû aux impuretés et calculé au $2^{\mathrm{e}}$ ordre (d'ailleurs divergent avec notre forme de $V$ ), et doit donc être éliminé comme nous l'avons expliqué plus haut. On peut effectuer l'intégrale qui subsiste seule dans (21) et on obtient

$$
\Delta(\omega)=-i \frac{q}{2 m l}
$$

où le nombre complexe $q(\omega)$ est défini par les conditions

$$
\begin{gathered}
\frac{q^{2}}{2 m}=\omega-\Delta(\omega) \\
I m q>0
\end{gathered}
$$

$l$ est le libre parcours moyen habituel :

$$
l=\frac{\pi}{m n V^{2}}
$$

Quand la partie imaginaire de $q$ peut être négligée, (22) est équivalent à la relation usuelle entre $l$ et le temps de collision $\tau$. $l=v \tau$ avec $\Delta=i / 2 \tau$. Toutefois, pour le problème qui nous intéresse il est nécessaire de garder les expressions rigoureuses (22) et (23) pour éviter certaines divergences.

En comprenant (22) et (23) on obtient l'équation pour $q(\omega)$

$$
q^{2}-\frac{i}{l} q-2 m \omega=0
$$

dont les racines sont

$$
q=\frac{i}{2 l} \pm \sqrt{2 \omega m-\frac{1}{4 l^{2}}} .
$$

Quand $\omega$ est réel négatif, il y a une seule racine de (26) qui satisfait à la condition $\left(23^{\prime}\right)$. Quand $\omega$ est réel et positif, les deux racines sont acceptables de ce point de vue. (Ceci correspond à l'existence d'ondes " entrantes " ou " sortantes " dans un problème de diffusion.) Le choix de la racine correcte, et donc de la valeur correcte de $\Delta(\omega)$ dans (19) est 
alors fixé par la condition classique : $G_{\omega}(k)$ considérée comme fonction de $\omega$, pour $k$ réel fixé, a un pôle $\omega=\Omega_{k}$. La partie imaginaire de $\Omega_{k}$ est $d u$ signe de $\left(k_{F}^{2}-k^{2}\right) / 2 m$. est

Dans le cas présent, l'équation explicite pour $\Omega_{k}$

$$
\begin{aligned}
\Omega_{k}-\Delta\left(\Omega_{k}\right) & =\frac{q^{2}}{2 m}\left(\Omega_{k}\right)=\frac{k^{2}}{2 m} \\
q\left(\Omega_{k}\right) & = \pm k
\end{aligned}
$$

et, d'après (25),

$$
\Omega_{k}=\frac{1}{2 m}\left(k^{2} \mp \frac{i k}{l}\right) .
$$

Considérons par exemple le cas $k<k_{F}$, alors nous imposons Im $\Omega_{k}>0$; nous devons prendre le signe - dans (27), et donc par comparaison le signe - dans (26). Dans la suite $q(\omega)$ représentera toujours la fonction définie par (26) qui a une partie imaginaire positive et qui satisfait au critère ci-dessus.

Pour terminer, nous citerons en vue d'applications ultérieures la forme spatiale obtenue en prenant la transformée de Fourier de (19)

$$
G_{\omega}\left(r r^{\prime}\right)=-\frac{m}{2 \pi\left|r-r^{\prime}\right|} \mathrm{e}^{i q\left|r-r^{\prime}\right|}
$$

4. Calcul du produit $\overline{G_{\omega} G_{\omega}}$ - Nous retournons maintenant à l'équation (15), en spécifiant que le potentiel $V(r)$ est purement local $V(r)=V \delta_{3}(r)$. Ceci permet d'écrire

$$
\overline{W(S) W(t)}=n V^{2} \delta_{3}(S-t)
$$

et de simplifier considérablement l'équation (15). Nous ne sommes intéressés en fait, d'après (14), qu'à la fonction $H_{\omega}(r)=F_{\omega}(r r)$ qui satisfait à l'équation

$H_{\omega}(r)=\overline{G_{\omega}(r 0)} \overline{G_{\omega}(0 r)}+n V^{2} \int \overline{G_{\omega}(r S)} \overline{G_{\omega}(S r)} H_{\omega}(S) \mathrm{d}_{3} S$

\section{Posons}

$$
K_{\omega}(p)=\int \overline{G_{\omega}(r 0)} \overline{G_{\omega}(0 r)} \text { eip. } r_{\mathrm{d}_{3}} r=\frac{m^{2}}{n p} \operatorname{tg}^{-1}\left(\frac{i p}{2 q(\omega)}\right)
$$

(où nous avons fait usage de (29)). Nous obtenons

$$
H_{\omega}(p)=\int H_{\omega}(r) \mathrm{e}^{i p . r} \mathrm{~d}_{3} r=\frac{K_{\omega}(p)}{1-n V^{2} K_{\omega}(p)} .
$$

5. Intégration sur les fréquences. - Il est commode de considérer $K_{\omega}(p)$ et $H_{\omega}(p)$ pour $p$ fixé comme des fonctions de la variable (complexe) $q$ plutôt que comme des fonctions de $\omega$. L'intégrale $\int \mathrm{d} \omega$ dans (14) peut être complétée par un grand demi-cercle dans le plan complexe des $\omega$
$(\operatorname{Im} \omega>0)$. Le parcours correspondant ABCDE dans le plan complexe des $q$ est représenté sur la figure 1. La relation entre les éléments différentiels se déduit de (25)

$$
\mathrm{d} \omega=(q-i \mid 2 l) \frac{\mathrm{d} q}{m} .
$$

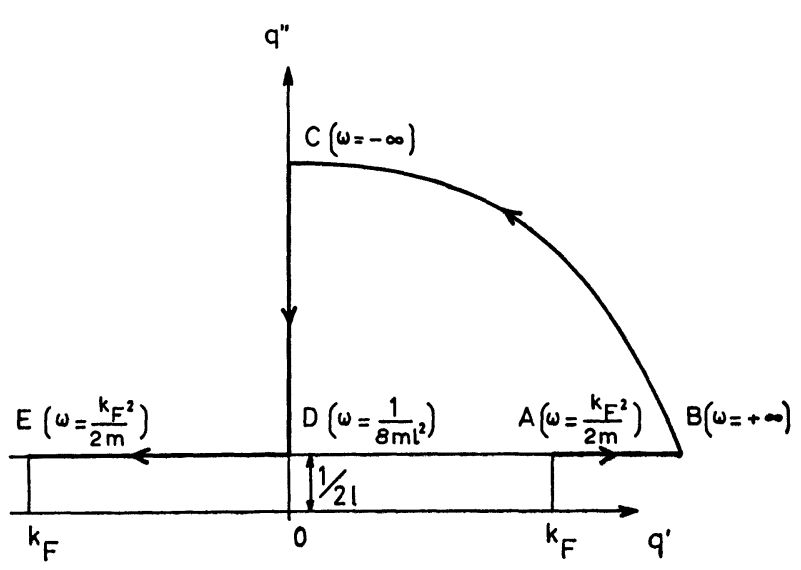

FIG. 1. - Parcours d'intégration de l'équation (14) dans le plan de la variable complexe $q$ définie par (26).

Nous allons maintenant remplacer le parcours ABCDE par le segment AE. Ceci est permis car $H_{\omega}(p)$ n'a pas de pôle dans le plan $(q)$ au-dessus de la ligne $q^{\prime \prime}=1 / 2 l$. Pour démontrer cette propriété, nous remarquons que $K_{\omega}(p)$ n'a pas de pôle dans le demi-plan $q^{\prime \prime}>0$ donc que les seuls pôles possibles pour $H_{\omega}(p)$ dans cette région correspondent à

$$
n V^{2} K_{\omega}(p)=1
$$

L'équation (35) admet bien une solution

$$
\begin{gathered}
q^{\prime}=0 \\
q^{\prime \prime}=\frac{p}{2 \operatorname{tg}(p l)}
\end{gathered}
$$

(lorsque $p l<\pi / 2$ ) mais le pôle (36) est en dessous de la ligne $q^{\prime \prime}=1 / 2 l$, donc la propriété est vérifiée.

Il est intéressant de remarquer que si l'on avait remplacé (14) par l'équation un peu moins bonne, mais plus simple :

$$
\begin{aligned}
G_{\omega}\left(r r^{\prime}\right)= & G_{\omega}^{0}\left(r r^{\prime}\right) \\
& +\int \mathrm{d}_{\mathbf{3}} S \mathrm{~d}_{\mathbf{3}} t G_{\omega}^{\mathbf{0}}(r S) \overline{W(S) W(t)} G_{\omega}^{\mathbf{0}}(S t) \overline{G_{\omega}\left(t r^{\prime}\right)}
\end{aligned}
$$

(qui est souvent suffisante pour traiter les effets de diffusion multiple $\left(14^{\prime}\right)$ ) le chemin d'intégration dans le plan $(q)$ traverserait le pôle (36) et une singularité non physique serait apparue.

En regroupant (4), (14), (33) et (34), et en effectuant l'intégration sur le segment $\mathrm{AB}$, nous obtenons finalement

$$
\chi(p)=\frac{m}{2 \pi^{2}} \int_{-k_{F}}^{k_{F}} q^{\prime} \mathrm{d} q^{\prime} \frac{S}{p-1 / 2 l S}
$$


avec

$$
\begin{aligned}
S & =S^{\prime}+i S^{\prime \prime} \\
S^{\prime} & =\operatorname{tg}^{-1}\left(\frac{2 q^{\prime}+p}{2 q^{\prime \prime}}\right)+\operatorname{tg}^{-1}\left(\frac{-2 q^{\prime}+p}{2 q^{\prime \prime}}\right) \\
S^{\prime \prime} & =\frac{1}{2} \log \frac{\left(2 q^{\prime}-p\right)^{2}-4 q^{\prime \prime 2}}{\left(2 q^{\prime}-p\right)^{2}+4 q^{\prime \prime 2}} \\
q^{\prime \prime} & =\frac{1}{2 l} .
\end{aligned}
$$

Dans (38) les $\operatorname{tg}^{-1}$ sont pris dans l'intervalle $(-\pi / 2, \pi / 2)$. On peut finalement écrire (37) sous la forme réelle

$$
\chi(p)=\frac{m p}{2 \pi^{2}} \int_{-k_{F}}^{k_{F}} q^{\prime} \mathrm{d} q^{\prime} \frac{S^{\prime \prime}}{\left(p-\frac{1}{2 l} S^{\prime}\right)^{2}+\left(\frac{S^{\prime \prime}}{2 l}\right)^{2}} .
$$

La formule $\left(39^{\prime}\right)$ se prête bien au calcul numérique. Dans le cas du métal pur $(l=\infty)$ on vérifie aisément qu'elle conduit à la forme habituelle

$$
\chi(p)=\frac{m k_{F}}{2 \pi^{2}}\left\{1+\frac{k_{F}}{p}\left(1-\frac{p^{2}}{4 k_{F}^{2}}\right) \log \left|\frac{p+2 k_{F}}{p-2 k_{F}}\right|\right\}
$$

\section{DISCUSSION.}

Les résultats numériques déduits de la formule (39) sont représentés sur les figures $2 a$ et $2 b$ utilisant les variables réduites

$$
\begin{gathered}
\frac{p}{k_{F}}=\lambda \\
\frac{1}{2 l k_{F}}=\mu \\
\chi(p)=J_{\mu}(\lambda) \frac{m k_{F}}{2 \pi^{2} \hbar^{2}} .
\end{gathered}
$$

On a représenté $J_{\mu}(\lambda)$ pour quelques valeurs typiques de $\mu$ (c'est-à-dıre du libre parcours moyen). Les conclusions principales sont les suivantes :

a) Quel que soit $\mu, J_{\mu}(0)$ est égal à 2 comme dans le métal pur. On vérifie ainsi la règle de somme (6).

b) $J_{\mu}(\lambda)$ diffère de $J_{\mu}(0)$ surtout dans la région $\lambda=2\left(p=2 k_{F}\right)$. Pour $\lambda=2 \mathrm{~d} J_{0} / \mathrm{d} \lambda(\lambda){ }^{*}$ a une singularité logarithmique (qui est à l'origine de l'anomalie de Kohn [7] dans le spectre des phonons longitudinaux). Dès que $\mu$ est différent de 0 , cette singularité disparaît. Il reste toutefois un «coude »

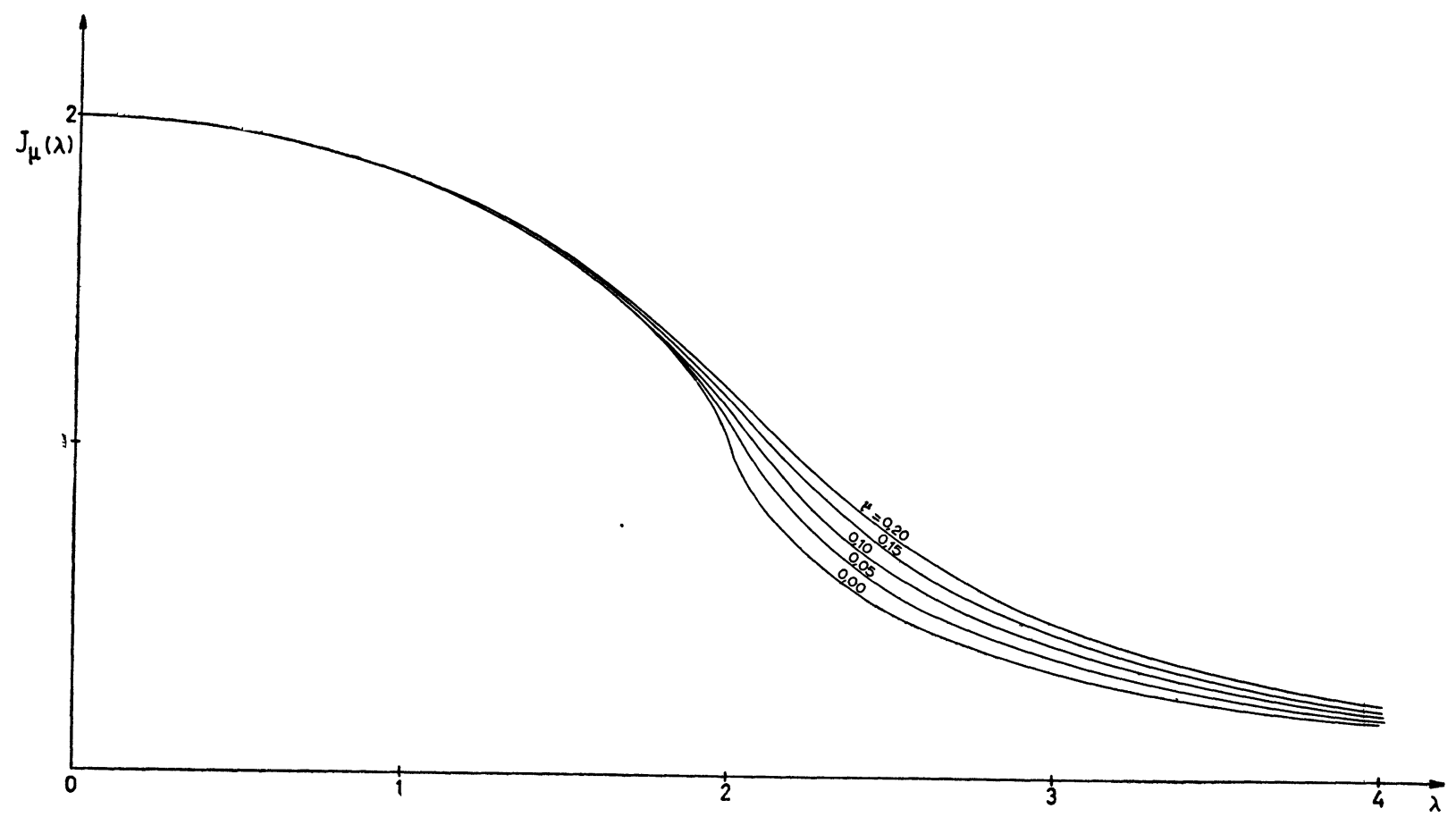

FIG. $2 a$. - Susceptibilité statique $\chi(p)$ d'un métal impur en fonction düvecteur d'onde $p$. VEn abscisse $\mu=p / k_{F}$. En ordonnée $J_{p}(\lambda)=\frac{2 \pi^{2} \hbar^{2}}{m k_{F}} \chi(p)$ pour différentes valeurs du libre parcours moyen_l caractérisé par $\mu=1 / 2 l k_{F}$.

observable dans le diagramme $J_{\mu}(\lambda)$ près de $\lambda=2$ tant que $\mu$ est inférieur à $1 \%$ environ. Cette région est représentée plus en détail sur la figure $2 b$. Elle a un certain intérêt en ce qui concerne l'observation éventuelle de l'anomalie de Kohn sur des métaux où le libre parcours moyen est limité, soit par des impuretés, soit par l'agitation thermique. (Dans ce deuxième cas toutefois il faudrait reprendre le calcul et tenir compte de l'aspect dynamique de la diffusion.) 
c) Aux grands $\lambda$, le comportement asymptotique de $J_{\mu}^{\prime}(\lambda)$ est donné par

$$
J_{\mu}(\lambda)=\frac{8}{3 \lambda^{2}}+0\left(\frac{1}{\lambda^{2}}\right)
$$

quelque soit $\mu$. Ceci revient à dire que la singularité de $\chi(r)$ pour $r \rightarrow 0$ n'est pas modifiée par les effets de libre parcours moyen, ce qui est assez naturel physiquement.

L'aspect de la transformée de Fourier $\chi(r)$ est représenté sur la figure 3 . On a posé

$$
\begin{aligned}
J_{\mu}(\rho) & =\frac{4 \pi}{\rho} \int_{0}^{\infty} \lambda d \lambda \sin \lambda_{\rho} J_{\mu}(\lambda) \\
\chi(r) & =\frac{m k_{F}^{4}}{16 \pi^{5} \hbar^{2}} J_{\mu}(\rho)
\end{aligned}
$$

Conformément à ce que nous avions annoncé dans la section I, la courbe $J_{\mu}(\rho)$ n'est pas seulement réduite en amplitude par rapport à $J_{0}(\rho)$, mais elle est fortement déformée. Cette déformation, qui dépend de $\mu$, peut avoir des effets importants dans les systèmes où 1) la partie longue distance des oscillations joue un rôle important, 2) le libre par-

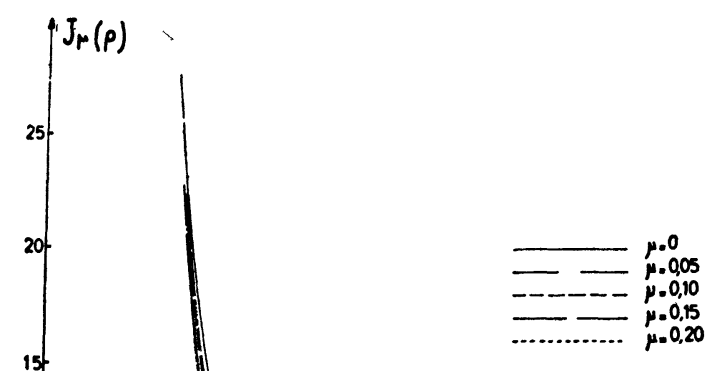

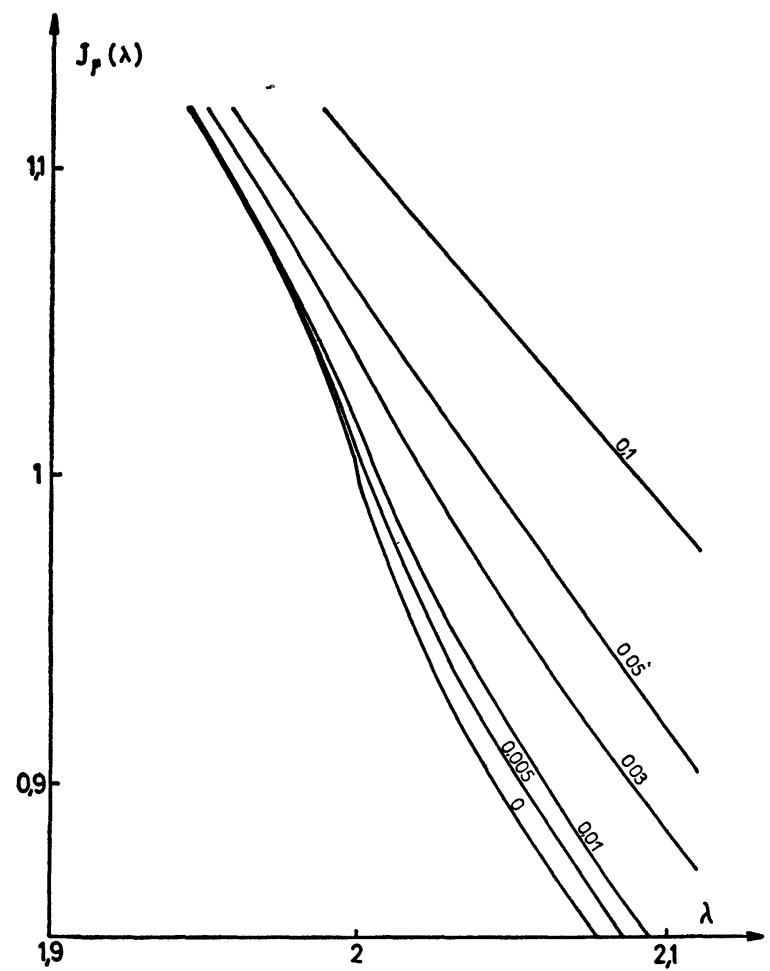

Fig. 2b. - Agrandissement de la région centrale de la figure précédente, montrant la disparition de l'anomalie de Kohn dans $\chi(p)$ en présence d'un libre parcours moyen fini. 
cours moyen varie fortement avec la température. Ainsi dans les alliages subissant une transformation

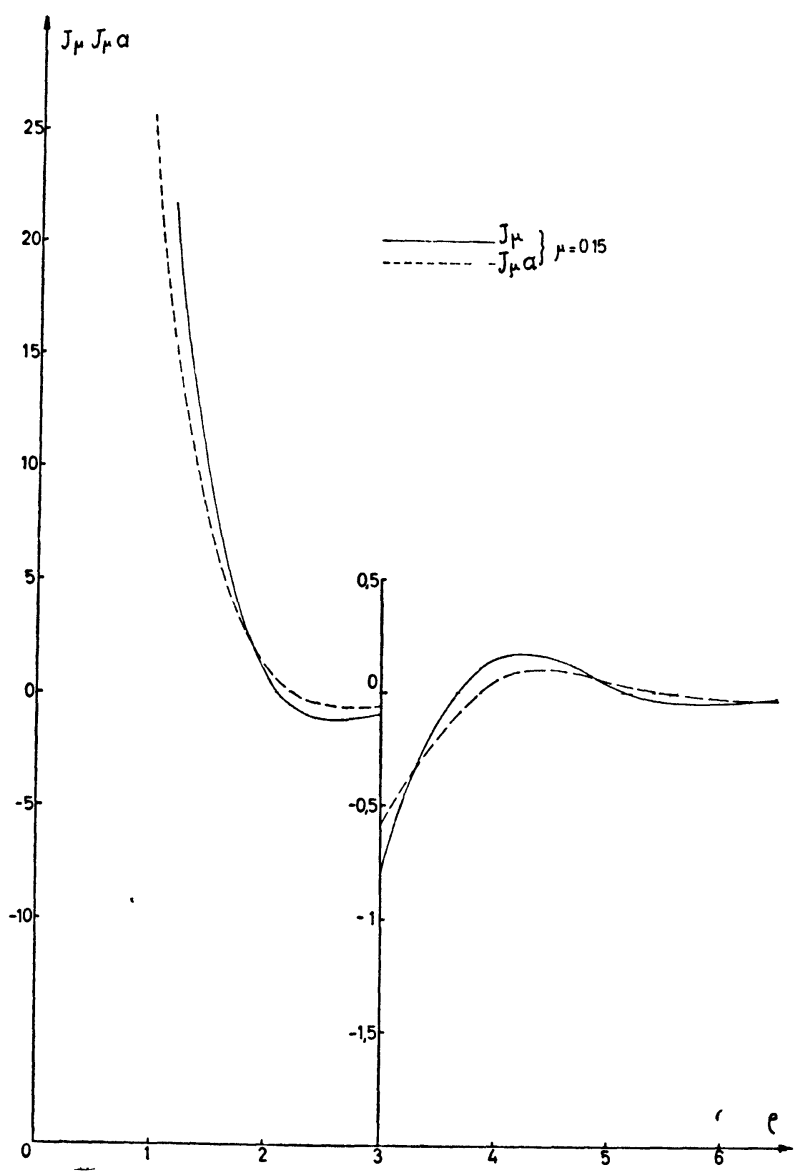

FIG. 4. - Comparaison entre la polarisation $J(\rho)$ calculée par la formule correcte (éq. 39 et 41) et la forme "intuitive mais incorrecte " $J a(\rho)$ (éq. 5). Noter la différence de phase entre les deux courbes. ordre désordre, l'interaction effective entre atomes, dont la dépendance spatiale est en gros proportionnelle à $J_{\mu}^{\prime}(\rho)$, dépend en fait de la température par l'intermédiaire de $\mu$. Ceci peut être important surtout pour les alliages formant des antiphases dans lesquelles les interactions entre seconds voisins (et aussi entre voisins plus éloignés) jouent un rôle important [8]. Un autre exemple est fourni par les métaux de terres räres, où les spins des électrons $4 f$ portés par les différents atomes sont couplés par une interaction indirecte qui est encore proportionnelle à $J_{\mu}(\rho)$ (en première approximation) [9]. On sait que dans ces métaux la longue portée et le caractère oscillant des couplages sont fréquemment à l'origine de configurations spirales des spins. Il est important de remarquer aussi que $\mu$ est relativement grand au-dessus $d u$ point d'ordre $T_{0}(\mu \sim 1 / 15$ dans $\mathrm{Gd}$ à la température ordinaire) mais que $\mu$ devient très petit aux températures $<T_{0} / 2$ où les spins $4 f$ sont alignés et ne diffusent plus les électrons [10]. Donc les interactions d'échange effectives varient nettement avec la température dans ces métaux. Ceci contribure probablement à la forte variation du pas des hélices avec la température qui est observée dans de nombreux cas [11]. Nous avons fait un calcul préliminaire de cet effet dans l'approximation des électrons libres; il montre que le pas des hélices peut être modifié par un facteur 2 lorsque $\mu$ passe de 0 à 0.1 . Toutefois, pour faire un calcul relativement quantitatif des effets que nous venons de décrire (pour les transitions ordre-désordre ou pour les métaux de terres rares) il faudra tenir compte, dans le calcul de $\chi\left(r r^{\prime}\right)$ du fait que les atomes diffusants ne sont pas répartis entièrement au hasard mais sont aux nœuds d'un réseau périodique.

Nous tenons à remercier tout particulièrement le Professeur Friedel pour plusieurs discussions relatives à ce problème et $M$. D. Pillard qui a effectué les calculs numériques.

\section{REFERENCES}

[1] Ruderman (M. A.) et Kittel (C.), Phys. Res., 1954, 96, 99.

[2] Nozis̀res (P.) et Pines (D.), Phys. Rev., 1958, 109, $741,762,1062$.

[3] Friedel (J.), Advances in Physics, 1954, 3, 446.

[4] On trouve une mise au point assez détaillée sur ces travaux dans Rickayzen, Cours de l'École d'Été de Bergen (C. Fronsdal, éditeur), 1961.

[5] Nous choisissons de calculer $\chi(r)$ à partir d'une perturbation indépendante du spin. Le calcul à partir d'une perturbation dépendante du spin donne exactement le même résultat, dans l'approximation où nous nous plaçons (interactions entre électrons négligées).
[6] Les équations (14) et (15) supposent que la correction au premier ordre $\bar{W}$ aux niveaux d'un électron a été éliminée, conformément aux prescriptions adoptées dans l'introduction.

[7] Kон (W.), Phys. Re». Letters, 1959, 2, 393.

[8] Villain (J.), même conférence.

[9] De Gennes (P. G.), C. R. Acad. Sc., 1958, 247, 1836.

[10] Friedel (J.) et de Gennes (P, G.), J. Phys. Chem. Sol., 1958, 4, 71 .

[11] De Gennes (P. G.), Colloque National de Magnétisme, Orsay, 1962 à paraître au Journal de Physique. 